\title{
Morphology, crystallization and rheological behavior in poly(butylene succinate)/cellulose nanocrystal nanocomposites fabricated by solution coagulation
}

\author{
Yi-Dong Li, Qian-Qian Fu, Ming Wang, Jian-Bing Zeng* \\ School of Chemistry and Chemical Engineering, Southwest University, Chongqing
}

400715, China

Corresponding author, Email: jbzeng@swu.edu.cn (Jian-Bing Zeng)

\begin{abstract}
Nanocomposites consisting of poly(butylene succinate) (PBS) and cellulose nanocrystals (CNC) were fabricated by solution coagulation method. Morphology analysis indicated that CNC dispersed well in PBS matrix and rheological analysis suggested that PBS and CNC showed strong interactions.
\end{abstract} Thermal analysis indicated that the nanocomposites showed slightly increased glass transition temperature, significantly enhanced crystallization temperature and different melting behavior, compared to neat PBS. Study on crystallization indicated that small loading of CNC could significantly increase overall crystallization rate of PBS, meanwhile the crystallization mechanism and crystal structure remained unchanged. The significant enhancement in overall crystallization was attributed to the increased nucleation ability by incorporation of well dispersed CNC nanoparticles. Tensile testing indicated that the tensile strength and modulus were gradually improved with increasing $\mathrm{CNC}$ content, while the elongation at break decreased and even brittle fracture occurred when the content of CNC increased to $1.0 \mathrm{wt} \%$.

Keywords: Poly(butylene succinate); Cellulose nanocrystal; Nanocomposite; 1 
Isothermal crystallization kinetics; Solution coagulation.

\section{Introduction}

Indiscriminate use of fossil-based and non-degradable polymers has brought about significant pollutions on the environment, which has led to a renewed and increased interest in polysaccharides like cellulose, starch and chitin (S. Wang, Lu, \& Zhang, 2016; Zeng, He, Li, \& Wang, 2012; Zeng, et al., 2011; Zhang, Wang, Zhao, \& Wang, 2012; Zhang, Zhang, Wang, Chen, \& Wang, 2009; Zhu, Dong, Wang, \& Wang, 2010). Their attraction comes from the annual renewability, easy availability and excellent biodegradability. Cellulose is the most abundant polysaccharide which is a structural polymer found in various plants, animals and even in some primeval organisms such as bacteria, fungi, algae and amoebas (Lin, Huang, Chang, Feng, \& Yu, 2011). Cellulose nanocrystal (CNC), obtained from partial acidolysis of cellulose, has many attractive intrinsic properties such as nanoscale dimensions, large surface area, high mechanical strength and modulus, thus has attracted a great deal of attentions in polymer nanocomposites (Habibi, Lucia, \& Rojas, 2010). In addition, CNC is also biodegradable and sustainable, which makes it an ideal reinforcing agent for biobased and biodegradable polyesters such as poly(lactic acid) (PLA) (Fortunati, Peltzer, et al., 2012; Kamal \& Khoshkava, 2015; Lin, Huang, et al., 2011; Miao \& Hamad, 2016), poly(butylene succinate) (PBS) (Hu, Lin, Chang, \& Huang, 2015; Lin, Chen, Hu, \&

Huang, 2015; Lin, Yu, Chang, Li, \& Huang, 2011), and poly(3-hydroxybutyrate-co-3-hydroxyvalerate) (PHBV) (Yu, Yan, \& Yao, 2014). 
It is well-known that the final properties of polymer nanocomposites depend strongly on the dispersion state of nanofillers (Du, Yang, Zhao, Wang, \& Zeng, 2016). Good dispersion of nanoparticles with very small amount would impart the matrix polymer with various significantly improved properties such as enhanced crystallization, reinforced mechanical performance, improved rheological properties (M. Wang, Deng, Du, Zhao, \& Zeng, 2015; Zeng, Hu, Wang, Zhang, \& Zeng, 2016; Zhao et al., 2016). Therefore, achieving good dispersion of nanoparticles is one of the key issues to fabricate polymer nanocomposites. The simplest way to incorporate pristine $\mathrm{CNC}$ into polymer matrix is casting-evaporation with water as a solvent or dispersion medium due to the hydrophilic nature of CNC (Habibi et al., 2010). This method is useful for the composite with water soluble or water dispersible polymer as the matrix, such as poly(vinyl alcohol) (Fortunati et al., 2013; Paralikara, Simonsen, \& Lombardi, 2008), water-borne polyurethane (Z. Gao et al., 2012; Santamaria-Echart et al., 2016), and natural rubber latex (Mariano, El Kissi, \& Dufresne, 2016). However, this method is unadoptable for biodegradable polyesters, due to their water insolubility and non-dispersibility.

Biodegradable polyesters are soluble in some organic solvents. Casting evaporation with organic solvent was also used in preparation of CNC reinforced biodegradable polyester nanocomposites (Fortunati, Armentano, et al., 2012; Fortunati, Peltzer, et al., 2012; Miao \& Hamad, 2016; Zhou, He, Jiang, \& He, 2015). PBS/CNC nanocomposites were fabricated by casting-evaporation of mixtures of PBS chloroform solution and $\mathrm{CNC}$ chloroform dispersion, which was prepared with aid of 
ultrasound (Lin, Yu, et al., 2011). It is worth noting that hydrophilic CNC dispersion in chloroform is not stable due to the different polarities. Therefore, aggregation of $\mathrm{CNC}$ was not inevitable during the long evaporation process. Acetylation would decrease of hydrophilicity of $\mathrm{CNC}$ and thus stabilize dispersion of $\mathrm{CNC}$ in organic solvent to effectively prevent aggregation during evaporation process. In this way, PLA/CNC and PBS/CNC nanocomposites were prepared (Hu et al., 2015; Lin, Huang, et al., 2011). However, acetylation would reduce the cost efficiency in CNC reinforced polymer nanocomposite. Furthermore, the loading of modified CNC was still relatively high (more than $1 \mathrm{wt} \%$ ) in order to achieve designed objective. Therefore, it is still a challenge to well disperse original $\mathrm{CNC}$ in biodegradable polyesters to fabricate bio-nanocomposites.

In contrast to casting-evaporation, solution coagulation provides an efficient way in preventing aggregation of nanoparticles, because long time evaporation is avoided. This method has been widely used in fabrication of polymer nanocomposites consisting of water insoluble polymers and water dispersible nanoparticles such as graphene oxide (Huang, Xu, Fan, Xu, \& Li, 2013), graphene and carbon nanotubes (M. Wang et al., 2015; Xu et al., 2010), and modified carbon nanotubes (Du et al., 2016; Zeng et al., 2016; Zhao et al., 2016). The electronic conductivity, crystallization, rheological, and mechanical properties of the related biodegradable polyesters were significantly improved with small addition of the corresponding nanoparticles, usually less than $1.0 \mathrm{wt} \%$. Although this method succeeded in fabricating polymer nanocomposite, it has not been employed in preparation of $\mathrm{CNC}$ reinforced 
biodegradable polyester nanocomposite. In addition, solution coagulation is an eco-friendlier method than casting-evaporation, since solvent evaporation is avoided.

In this study, we evaluate the feasibility of solution coagulation in fabrication of original $\mathrm{CNC}$ reinforced PBS nanocomposites. It was reported that original $\mathrm{CNC}$ was dispersible in $\mathrm{N}, \mathrm{N}$-dimethyl formamide (DMF) and the dispersion was as stable as in aqueous dispersion (Samir, Alloin, Sanchez, El Kissi, \& Dufresne, 2004). Therefore, we use DMF as a solvent for PBS to mix with CNC water dispersion firstly and then use excess water as coagulation agent for the well-dispersed PBS/CNC dispersion to coagulate the PBS/CNC nanocomposites. $\mathrm{CNC}$ with various loadings from 0.1 to 1.0 wt\% was incorporated into PBS. The dispersion of $\mathrm{CNC}$ in the composites was investigated by scanning electron microscope. The crystallization behavior of the PBS nanocomposites with small addition of original CNC was studied with differential scanning calorimetry, polarized optical microscope, and X-ray diffraction in detail. In addition, the rheological and mechanical properties of the PBS/CNC nanocomposites were also investigated. To our knowledge, no literature has been reported on the fabrication of PBS/CNC nanocomposites by solution coagulation and on the investigation of morphology and crystallization in small amount CNC reinforced PBS nanocomposites.

\section{Experimental section}

\subsection{Materials}

Cellulose nanocrystals (CNC) water dispersion with concentration of 1.0 wt $\%$ was 
provided by Haojia nanocellulose science and technology Ltd. (Tianjin, China). It was prepared by acidolysis of cotton pulp. The average diameter and length were 7-10 and $200 \mathrm{~nm}$, respectively. Poly(butylene succinate) (PBS) with molecular weight of $1.2 \times 10^{5} \mathrm{~g} / \mathrm{mol}$ was obtained from Anqing Hexing Chemical Co., LTD (Anhui, China). $\mathrm{N}$, N-dimethyl formamide (DMF) was procured from Kelong Chemical Co., LTD (Chengdu, China). All the materials were used without further purification.

\subsection{Fabrication of CNC filled PBS nanocomposites}

PBS/CNC nanocomposites with CNC contents various from 0.1 to 1.0 wt $\%$ were prepared by solution coagulation method with DMF as solvent for PBS and water as CNC dispersion medium and coagulating agent, respectively. Taking a nanocomposite containing $0.1 \mathrm{wt} \% \mathrm{CNC}$ as an example, the detailed procedures are as follows: dissolving $14.98 \mathrm{~g}$ PBS in $200 \mathrm{~mL}$ DMF by magnetic stirring at $80{ }^{\circ} \mathrm{C}$ for $1 \mathrm{~h}$, then dropping $2 \mathrm{~mL} \mathrm{CNC}$ water dispersion into the magnetically stirred PBS solution, after mixing for $10 \mathrm{~min}$, adding excessive deionized water into the solution to precipitate the coagulated composites. Four PBS/CNC nanocomposites containing 0.1, 0.3, 0.5 and $1.0 \mathrm{wt} \% \mathrm{CNC}$ were prepared and named after PBS/CNC-0.1, PBS/CNC-0.3, PBS/CNC-0.5 and PBS/CNC-1.0, respectively. Neat PBS was also treated with the same procedure for property comparison. The samples were collected by filtration and vacuum dried at $60{ }^{\circ} \mathrm{C}$ for 2 days. Then, the sample sheets with thickness of $1 \mathrm{~mm}$ were prepared by compression molding at $130{ }^{\circ} \mathrm{C}$ under $10 \mathrm{MPa}$ pressure for further characterization. 


\subsection{Characterization}

Morphology of PBS/CNC nanocomposites was observed on a XL-30s FEG (Philips, Holland) scanning electron microscope (SEM) with an accelerating voltage of $5 \mathrm{kV}$. The cryo-fractured surfaces were used for observation and were sputtered with a layer of gold prior to measurement.

Rheological behavior of neat PBS and PBS/CNC composites was tested on a TA DHR-1 rotational rheometer in dynamic frequency sweep mode from 0.1 to $100 \mathrm{rad} / \mathrm{s}$ at $140{ }^{\circ} \mathrm{C}$ with an oscillation strain of $1.0 \%$.

Thermal property and non-isothermal crystallization of neat PBS and PBS/CNC nanocomposites were measured by a NETZSCH differential scanning calorimeter (DSC-214). About $6 \mathrm{mg}$ sample sealed in aluminum pan was first heated to $140{ }^{\circ} \mathrm{C}$ and kept at this temperature for $3 \mathrm{~min}$ to remove thermal history, then cooled to $-60{ }^{\circ} \mathrm{C}$ at a cooling rate of $10{ }^{\circ} \mathrm{C} / \mathrm{min}$, and finally reheated to $140{ }^{\circ} \mathrm{C}$ at the same rate. The measurement was performed under $\mathrm{N}_{2}$ atmosphere. Both the cooling and the second heating scans were recorded for data analysis.

Isothermal crystallization kinetics of neat PBS and PBS/CNC nanocomposites was carried out on the NETZSCH DSC-214 equipment. About $6 \mathrm{mg}$ sample sealed in aluminum pan was first melted at $140{ }^{\circ} \mathrm{C}$ for $3 \mathrm{~min}$ to remove thermal history, and then quickly cooled to $80{ }^{\circ} \mathrm{C}$ at a cooling rate of $60{ }^{\circ} \mathrm{C} / \mathrm{min}$, and finally kept at the temperature until crystallization finished. All measurements were carried out under $\mathrm{N}_{2}$ atmosphere. The exothermic curves were recorded for analysis. 
Spherulitic morphology of neat PBS and PBS/CNC nanocomposites was studied on an Olympus BX51 polarized optical microscope (POM) with a RT600 temperature controller. The sample film between two microscopic cover glasses was first melted at $140{ }^{\circ} \mathrm{C}$ for 3 min to diminish thermal history, and then quickly quenched to $80{ }^{\circ} \mathrm{C}$ and kept at this temperature until crystallization finished.

X-ray diffraction (XRD) measurement was performed on a Philips X'Pert X-ray diffractometer with $\mathrm{Cu} \mathrm{K \alpha}$ radiation and a scan rate of $2 \% / \mathrm{min}$ from $5^{\circ}$ to $40^{\circ}$ at room temperature.

Mechanical property of neat PBS and PBS/CNC nanocomposites was measured on a Sansi Universal Testing Machine (CMT6503) at a crosshead speed of $20 \mathrm{~mm} / \mathrm{min}$ at room temperature in general accordance with ISO 527-3. Dumbbell-shaped specimen with width and thickness of 4 and $1 \mathrm{~mm}$ was cut from the sample sheets and used for tensile testing. The gauge length between the grips was $25 \mathrm{~mm}$. Five specimens were measured for each sample, and average result was reported for all samples.

\section{Results and Discussion}

\subsection{Morphology of PBS/CNC nanocomposites}

To evaluate the feasibility of solution coagulation method in fabrication CNC filled biodegradable polyester nanocomposite, the morphologies of the prepared PBS/CNC nanocomposites were studied by SEM. Fig. 1 shows the SEM images for the cryo-fractured surfaces of neat PBS and PBS/CNC nanocomposites with different CNC loadings. Neat PBS showed a smooth surface as shown in Fig. 1a. PBS/CNC 
nanocomposites showed rough surfaces, and the roughness increased with increasing CNC loading. CNC nanoparticles, the bright dots directed by blue arrows, dispersed very well in PBS/CNC nanocomposites. No aggregation was detected on surface even with CNC loadings increased to $1.0 \mathrm{wt} \%$, as shown in Fig. 1e. In addition, there was no pulling out of $\mathrm{CNC}$, indicating a strong interfacial adhesion between the dispersed CNC and the PBS matrix. As CNC did not pulling out, it had to break when suffering from external forces, which would induce deformation of the surrounding matrix. With content of CNC increased, more significant matrix deformation occurred, which accounted for the increased roughness for the surface of PBS/CNC composite with increasing content of $\mathrm{CNC}$.
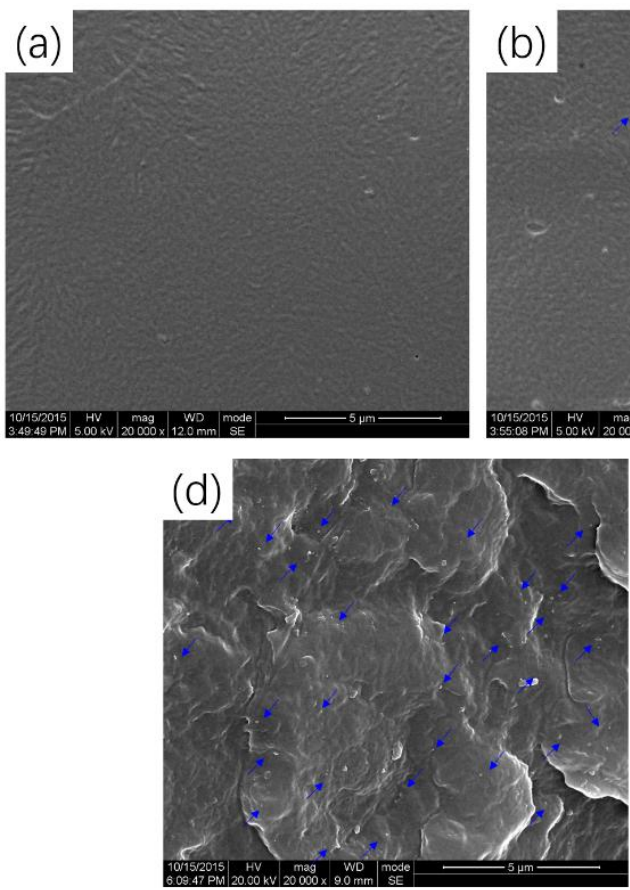
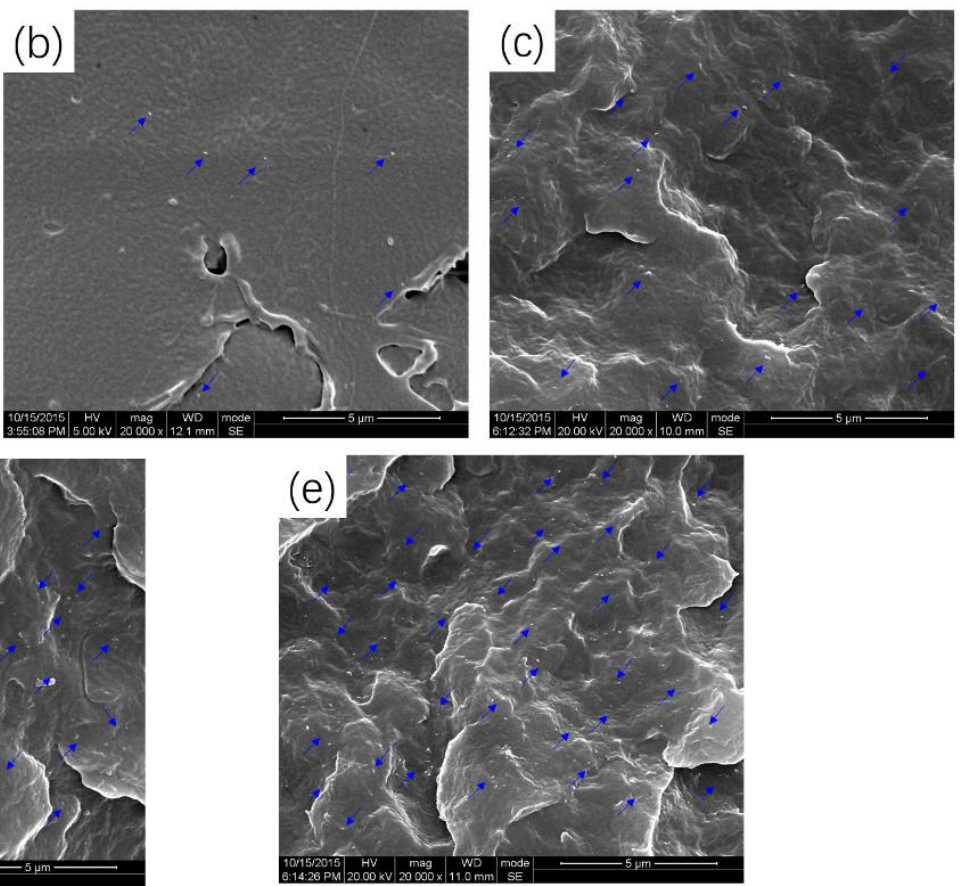

Fig. 1. SEM images for the cryo-fractured surfaces of neat PBS (a), PBS/CNC-0.1 (b), PBS/CNC-0.3 (c), PBS/CNC-0.5 (d) and PBS/CNC-1.0 (e). 


\subsection{Rheological behavior}

Rheology is a very useful technology to analyze the microscopic structures of polymer composites, because the rheological parameters are highly respected to the dispersion state of fillers and the interactions between fillers and matrix (Du et al., 2016; C. Gao et al., 2014; Yuan, Wu, Zhang, Zhou, \& Lin, 2011). To study the effect of $\mathrm{CNC}$ on the rheological behavior of PBS nanocomposites, the frequency sweeps were performed at $140{ }^{\circ} \mathrm{C}$ from 0.1 to $100 \mathrm{rad} / \mathrm{s}$. Fig. 2 shows the dependence of storage modulus (G') and complex viscosity $\left(\left|\eta^{*}\right|\right)$ on angular frequency $(\omega)$ for neat PBS and PBS/CNC nanocomposites.
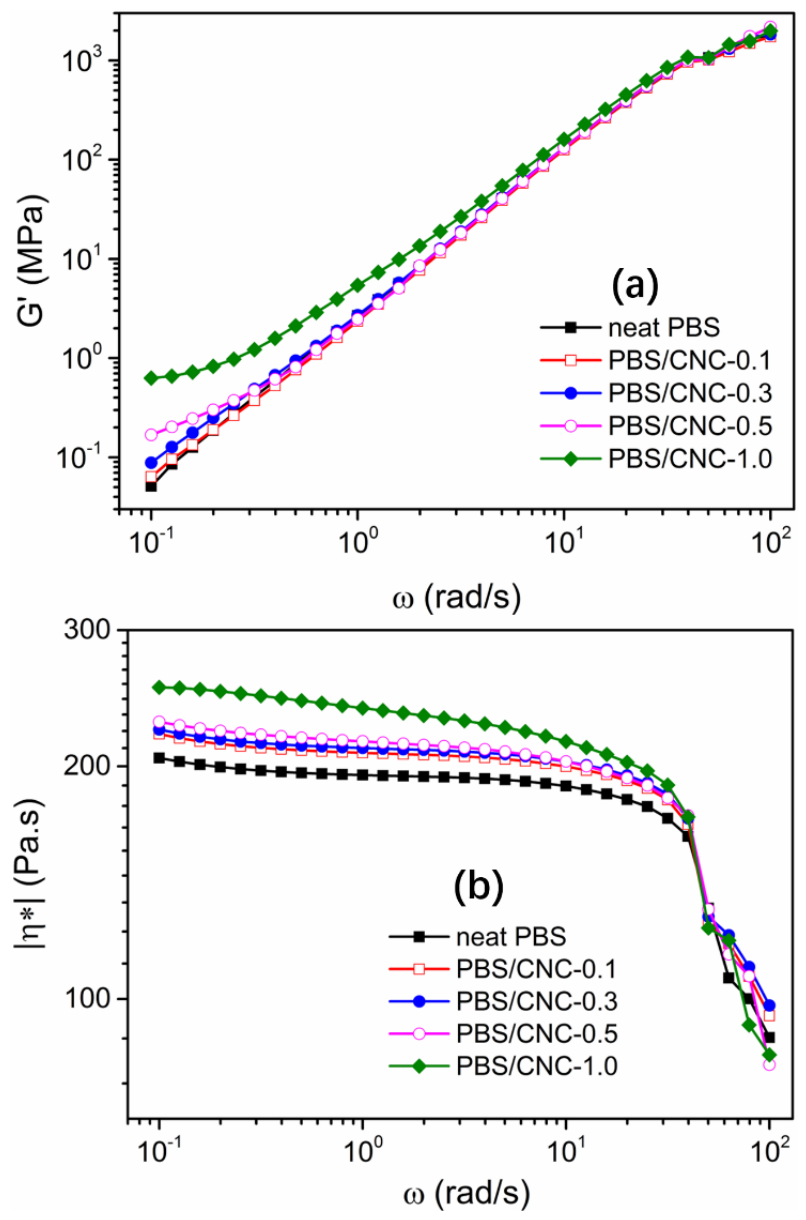

Fig. 2. Storage modulus G' (a) and complex viscosity $\left|\eta^{*}\right|$ (b) of neat PBS and 
$\mathrm{PBS} / \mathrm{CNC}$ nanocomposites as a function of angular frequency at $140{ }^{\circ} \mathrm{C}$.

It is obvious from Fig. 2a that neat PBS showed terminal behavior at low $\omega$, indicating a liquid-like response. The terminal behavior decreased with increasing content of CNC. When the content of $\mathrm{CNC}$ was $0.5 \mathrm{wt} \%$, the G' plots deviated apparently from terminal behavior at low frequency, indicating increased solid-like response. With further increasing the content of $\mathrm{CNC}$ to $1.0 \mathrm{wt} \%$, the deviation become more pronounced and extended to higher frequency. Furthermore, an obvious plateau was observed at low frequency, indicating the formation of network structure of CNC in PBS matrix. Similar results were also observed in some other nanoparticles reinforced PBS nanocomposites (Yuan et al., 2011; Zeng et al., 2016). Complex viscosity plots, as shown in Fig. 2b, indicated that all samples showed Newton plateau at low frequency, while shear thinning was pronounced at high frequency. It is noted that the complex viscosity increased gradually with the CNC content, indicating a strong interaction between CNC and PBS matrix (Mishra, Hwang, \& Ha, 2005). The effect of $\mathrm{CNC}$ is more pronounced at low $\omega$ and the relative effect reduced with increasing frequency due to shear thinning (Pötschke, Fornes, \& Paul, 2002).

\subsection{Thermal transition and non-isothermal crystallization}

Strong interfacial adhesion between the polymer matrix and the well dispersed nanoparticles would obviously influence the thermal transition and crystallization behavior of the matrix polymer. Therefore, we studied the effect of CNC content on 
the thermal transition and non-isothermal crystallization behavior of PBS by DSC. Fig. 3 shows the DSC cooling and the second heating scans of neat PBS and PBS/CNC nanocomposites.
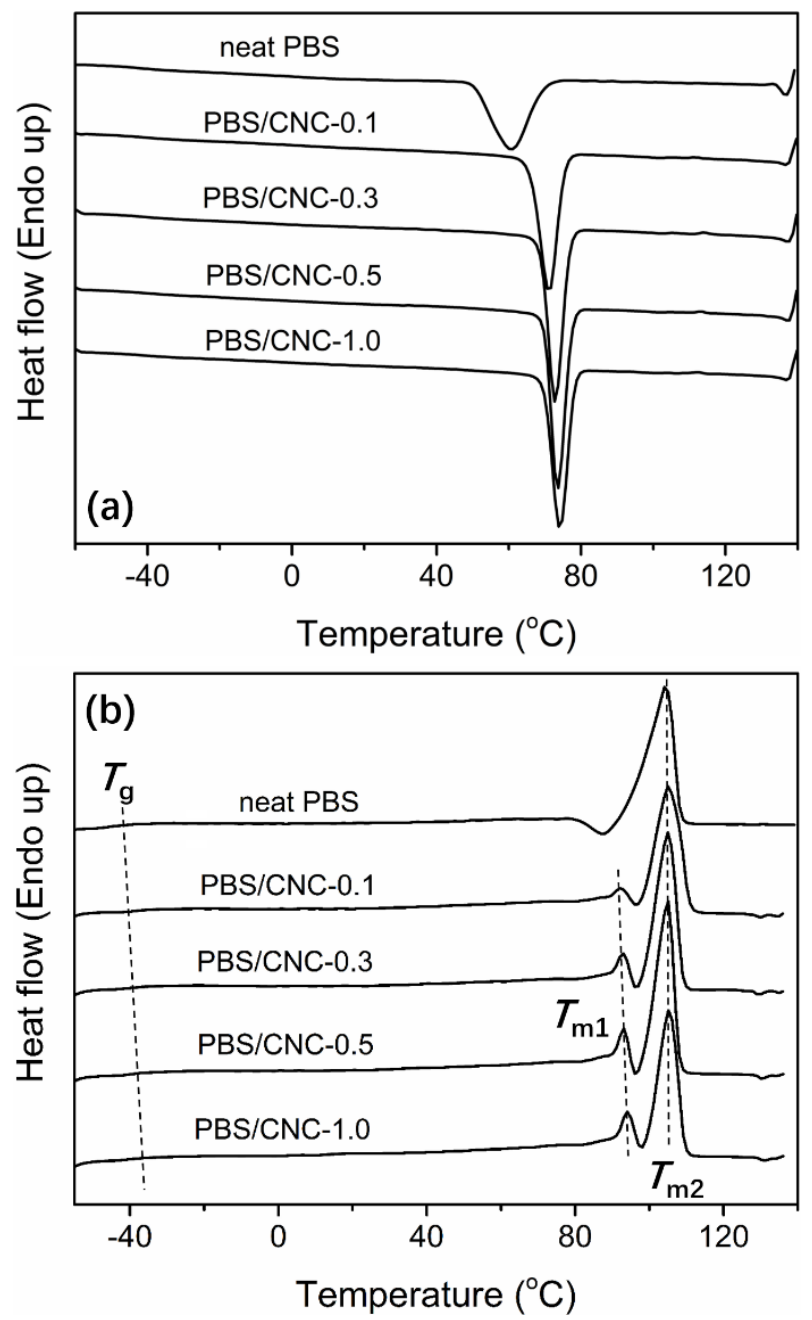

Fig. 3. DSC cooling (a) and the second heating scans (b) of neat PBS and PBS/CNC nanocomposites.

It is seen from Fig. 3a that the non-isothermal crystallization of neat PBS showed a relatively wide exothermic peak with the crystallization peak temperature $\left(T_{\mathrm{c}}\right)$ of $59.6{ }^{\circ} \mathrm{C}$. After incorporation of $\mathrm{CNC}$, the exothermic non-isothermal crystallization 
peak narrowed significantly and the crystallization peak temperature shifted to higher temperatures with increasing $\mathrm{CNC}$ content. With addition of only $0.1 \mathrm{wt} \% \mathrm{CNC}$, the $T_{\mathrm{c}}$ of PBS increased considerably to $71.1^{\circ} \mathrm{C}$. With further increasing $\mathrm{CNC}$ content, the $T_{\mathrm{c}}$ gradually increased to $72.8,73.5$ and $74.1{ }^{\circ} \mathrm{C}$ for PBS/CNC-0.3, PBS/CNC-0.5 and PBS/CNC-1.0, respectively. This change indicated that incorporation of well dispersed CNC could enhance crystallization rate of PBS significantly even with very small addition, possibly due to the nucleation effect of well dispersed CNC nanoparticles. It was noted that the crystallization enthalpies for all samples were $\sim 60$ $\mathrm{J} / \mathrm{g}$, indicating incorporation of $\mathrm{CNC}$ only accelerated the crystallization rate but did not change the degree of crystallinity. It was interesting to find from Fig. $3 b$ that the glass transition temperature $\left(T_{\mathrm{g}}\right)$ of PBS increased slightly to higher temperatures with increasing $\mathrm{CNC}$ content, which was attributed to the decreased chain mobility of PBS resulted from the strong interaction between dispersed CNC and PBS matrix. The value of $T_{\mathrm{g}}$ was $-40.2,-39.5,-38.9,-39.0$ and $-38.6{ }^{\circ} \mathrm{C}$ for neat PBS, PBS/CNC-0.1, PBS/CNC-0.3, PBS/CNC-0.5 and PBS/CNC-1.0, respectively. Neat PBS showed a single melting peak with a small crystallization peak before melting, which was ascribed to the melt-recrystallization of crystals with low thermal stability (Qiu, Komura, Ikehara, \& Nishi, 2003; Zeng, Srinivansan, Li, Narayan, \& Wang, 2011). It was interesting to find that PBS/CNC nanocomposites showed double melting peaks with one minor peak lower than $100{ }^{\circ} \mathrm{C}$ and the other major peak higher than $100{ }^{\circ} \mathrm{C}$. The peak temperature of the minor melting peak $\left(T_{\mathrm{m} 1}\right)$ increased gradually to higher temperatures while that of the major melting peak $\left(T_{\mathrm{m} 2}\right)$ kept unchanged at $105{ }^{\circ} \mathrm{C}$ 
with increasing CNC content. The fusion enthalpy of neat PBS and PBS/CNC nanocomposites was all around $61 \mathrm{~J} / \mathrm{g}$, which was in agreement with the crystallization enthalpy.

The double melting peaks could also be explained by the melt-recrystallization mechanism. Previous investigations indicated that small and/or imperfect crystals shift successively to more stable crystals during melt-recrystallization, which means melting and recrystallization are competitive under heating process (Yasuniwa, Tsubakihara, Ohoshita, \& Tokudome, 2001). Accordingly, endothermic peak comes out when melting rate surpasses recrystallization rate, while exothermic peak appears if recrystallization rate overwhelms melting rate. Investigation on melting behavior of PBS after non-isothermal crystallization at different cooling rates or isothermal crystallization at different temperatures by Satou and coworkers indicated that exothermic peak shifted gradually to endothermic peak with decreasing cooling rate or increasing isothermal crystallization temperature (Yasuniwa \& Satou, 2002; Yasuniwa, Tsubakihara, Satou, \& Iura, 2005). The crystals obtained under lower cooling rate or higher isothermal crystallization temperature are more stable since they have more perfect structure due to the formation at high temperatures. More stable crystals melted slowly, thus the recrystallization of melted crystals have to occur at higher temperatures. The crystallization rate decreased at such high temperatures. Therefore, melting rate is faster than crystallization rate and thus endothermic peak appears. This theory is also applicable to explain the double melting behavior of PBS/CNC nanocomposites. Since the crystallization of PBS 
occurs at higher temperatures with increasing $\mathrm{CNC}$ content, which would result in the formation of more stable crystals, thus reduce the recrystallization rate during heating process to exhibit endothermic peak.

\subsection{Isothermal crystallization behavior and crystal structures}

In order to study the effect of CNC with small addition on the crystallization of PBS in detail, the isothermal crystallization kinetics of neat PBS and PBS/CNC nanocomposites were comparatively investigated by DSC.
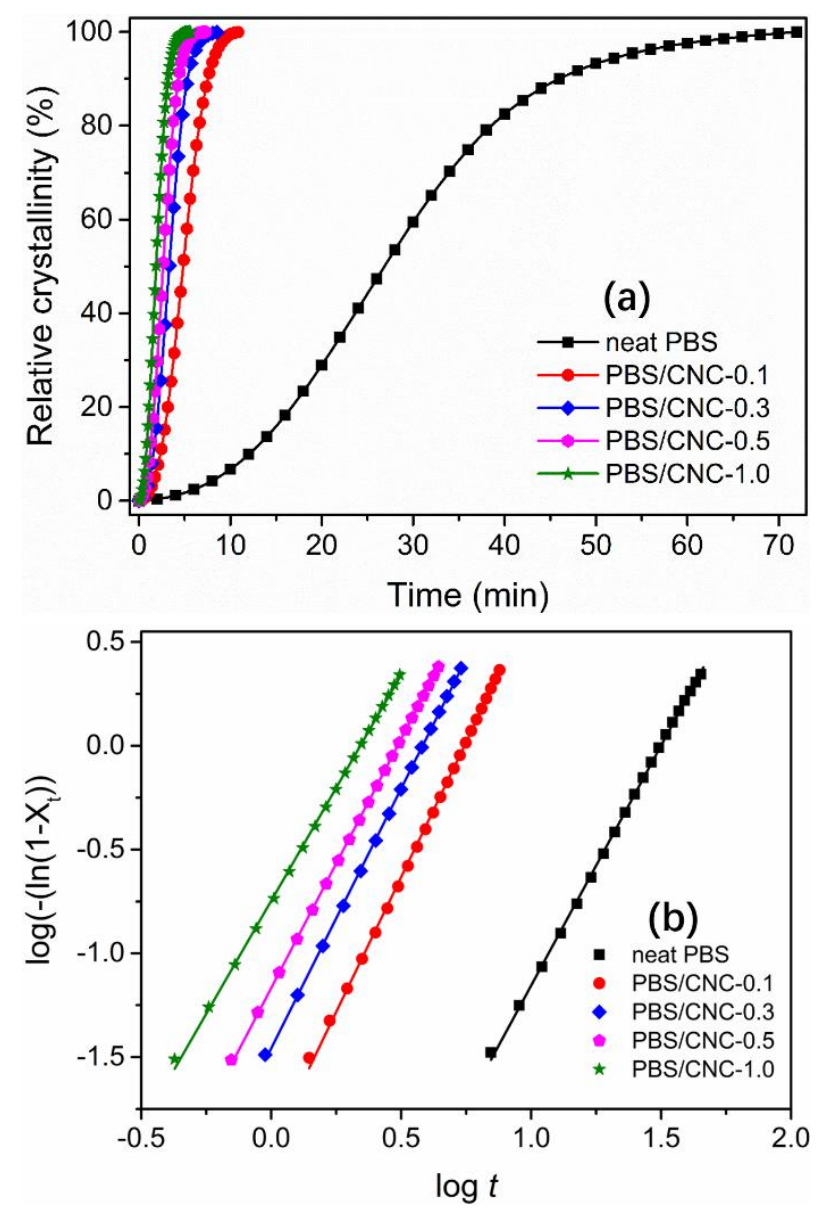

Fig. 4. (a) Development of relative crystallinity $\left(X_{\mathrm{t}}\right)$ with time $(t)$ for isothermal crystallization of neat PBS and PBS/CNC nanocomposites at $80{ }^{\circ} \mathrm{C}$ and (b) Avrami 
plots of neat $\mathrm{PBS}$ and $\mathrm{PBS} / \mathrm{CNC}$ nanocomposites at $80^{\circ} \mathrm{C}$.

Fig. 4a shows the development of relative crystallinity $\left(X_{c}\right)$ with time for isothermal crystallization of neat $\mathrm{PBS}$ and $\mathrm{PBS} / \mathrm{CNC}$ nanocomposites at crystallization temperature of $80{ }^{\circ} \mathrm{C}$. All plots showed similar sigmoidal shapes. Neat PBS finished crystallization in a long time of about $70 \mathrm{~min}$, while All PBS/CNC nanocomposites completed crystallization in a time of less than $10 \mathrm{~min}$. For the effect of $\mathrm{CNC}$ content, the time required for the composites to finish crystallization decreased with increasing $\mathrm{CNC}$ content. The results indicated small addition of $\mathrm{CNC}$ nanoparticles was efficient in accelerating crystallization rate of PBS.

The well-known Avrami method was employed to analyze the isothermal crystallization kinetics of neat PBS and PBS/CNC nanocomposites. The method supposes that the relationship of relative crystallinity $\left(X_{\mathrm{t}}\right)$ and crystallization time $(t)$ follows the equation:

$$
1-X_{t}=\exp \left(-\mathrm{k} t^{n}\right)
$$

where $X_{\mathrm{t}}$ and k represent relative crystallinity at a given time $\mathrm{t}$ and a rate constant, respectively. $\mathrm{n}$ is the Avrami exponent, which indicates the nature of the nucleation and growth process (Avrami, 1939). Double logarithm changes the Avrami equation to

$$
\log \left(-\ln \left(1-X_{t}\right)\right)=\log k+n \log t
$$

A straight line is obtained by plotting $\log \left(-\ln \left(1-X_{\mathrm{t}}\right)\right)$ with $\log t$, and the rate constant and the Avrami exponent can be deduced from the intercept and slope of plotting straight line, respectively. Fig. $4 \mathrm{~b}$ shows the Avrami plots of neat PBS and PBS/CNC 
nanocomposites by isothermal crystallization at $80{ }^{\circ} \mathrm{C}$. Almost five parallel straight lines were obtained, indicating that the Avrami equation was applicable to describe the isothermal crystallization kinetics of neat PBS and PBS/CNC nanocomposites. Accordingly, the values of $k$ and $n$ for neat PBS and PBS/CNC nanocomposites were calculated. All samples showed $n$ in the range of 2.0 3.0, and the values were 2.31, 2.60, 2.50, 2.39 and 2.18 for neat PBS, PBS/CNC-0.1, PBS/CNC-0.3, PBS/CNC-0.5 and PBS/CNC-1.0, respectively. The results indicate that incorporation of small amount of CNC would not change the isothermal crystallization mechanism of PBS, and they all followed a mechanism of three dimensional truncated spherulitic growth with athermal nucleation (Lu, Zeng, Huang, \& Wang, 2012; Zeng, Zhu, Li, Qiu, \& Wang, 2010). The $k$ value for neat PBS is $3.4 \times 10^{-4} \mathrm{~min}^{-\mathrm{n}}$. The values were $1.17 \times 10^{-2}$, $3.48 \times 10^{-2}, 6.8 \times 10^{-2}$ and $0.18 \mathrm{~min}^{-\mathrm{n}}$ for PBS/CNC-0.1, PBS/CNC-0.3, PBS/CNC-0.5 and PBS/CNC-1.0, respectively. Although the rate constant increased considerably with incorporation of $\mathrm{CNC}$, it is not suitable to compare the overall crystallization rate of the sample directly from the $k$ values, due to the variation in $n$ values of the samples. Therefore, crystallization half-life time $\left(t_{0.5}\right)$, the time required to achieve relative crystallinity of $50 \%$, was introduced to analyze isothermal crystallization kinetics. The reciprocal of $t_{0.5}$, i.e., $1 / t_{0.5}$, was usually used directly to describe the overall crystallization rate of samples (Liu et al., 2013; Song \& Qiu, 2011; Wu, Huang, Zeng, Li, \& Wang, 2014a). The value of $t_{0.5}$ was calculated by

$$
t_{0.5}=(\ln 2 / k)^{1 / n}
$$

The overall crystallization rate of neat PBS at $80{ }^{\circ} \mathrm{C}$ was $3.69 \times 10^{-2} \mathrm{~min}^{-1}$. The rate 
was increased by 5.6 times to $0.21 \mathrm{~min}^{-1}$ after incorporation of only $0.1 \mathrm{wt} \% \mathrm{CNC}$, and it was increased by $8.2,10.3$ and 14.5 times with further increasing CNC content to $0.3,0.5$ and $1.0 \mathrm{wt} \%$, respectively. The drastic enhancement in overall crystallization rate of PBS should be reasonably attributed to the increased nucleating ability by addition of CNC nanoparticles. The high specific surface area and large aspect ratio of well dispersed $\mathrm{CNC}$ nanoparticles would lower the surface free energy barrier towards nucleation to significantly accelerate nucleation and overall crystallization kinetics (Huang et al., 2013).
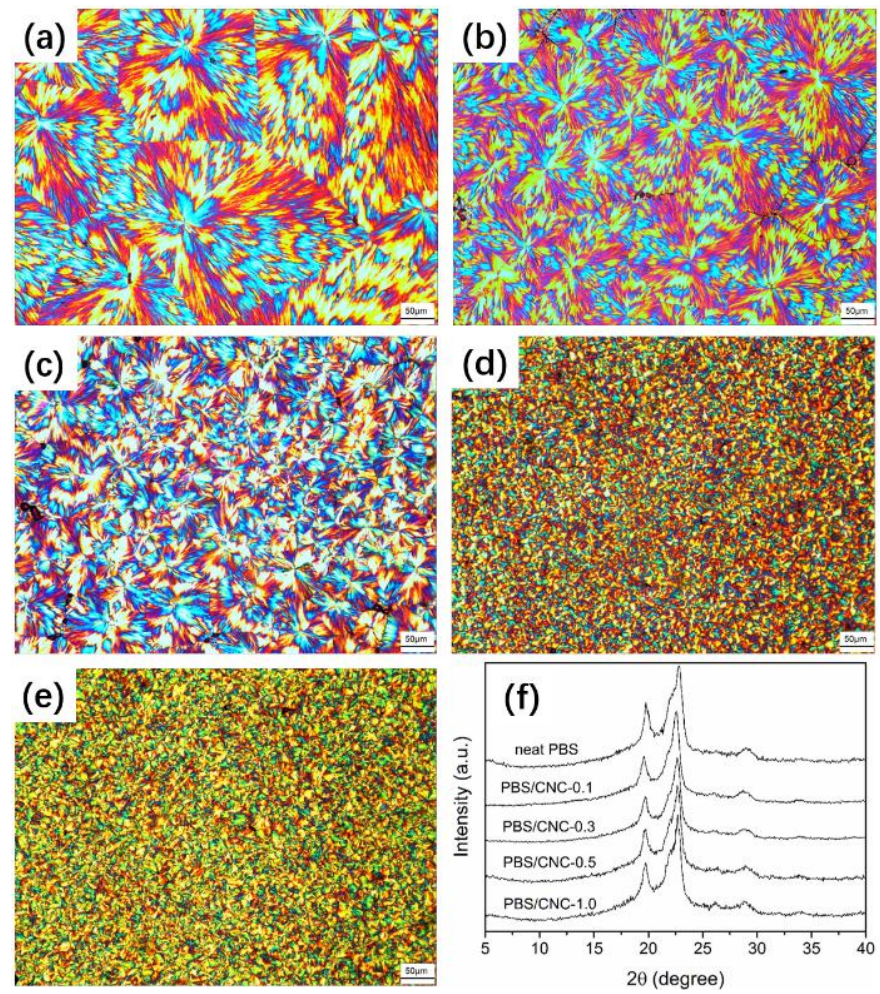

Fig. 5. Spherulitic morphology of neat PBS (a), PBS/CNC-0.1 (b), PBS/CNC-0.3 (c), PBS/CNC-0.5 (d), and PBS/CNC-1.0 (e) formed by isothermal crystallization at $80{ }^{\circ} \mathrm{C}, \mathrm{XRD}$ patterns of neat PBS and PBS/CNC nanocomposites (f). 
In order to observe the effect of $\mathrm{CNC}$ on nucleating density and crystalline morphology of PBS, the spherulitic morphology of neat PBS and PBS/CNC nanocomposites was recorded by $\mathrm{POM}$ after isothermal crystallization at $80{ }^{\circ} \mathrm{C}$, as shown in Fig. 5. PBS formed well developed and large spherulites with clear boundaries (Fig. 5a). With addition of $0.1 \mathrm{wt} \% \mathrm{CNC}$, the diameter of spherulites decreased significantly while the number of spherulites increased apparently as shown in Fig. 5b, indicating significantly increased nucleation density. With increase in CNC content, the size of spherulites decreased gradually and the nucleation density increased drastically. When the content of CNC increased to 0.5 (Fig. 5d) and $1.0 \mathrm{wt} \%$ (Fig. 5e), spherulitic boundaries were hard to discriminate, due to the rather high nucleation density. The results confirmed that well dispersed CNC worked as a very efficient nucleating agent for PBS.

XRD was performed to study the effect of CNC on the crystal structure of PBS. Fig. 5f shows the XRD patterns of neat PBS and PBS/CNC nanocomposites. Neat PBS shows three main characteristic diffraction peaks at $2 \theta$ of $19.7,21.9$ and $22.6^{\circ}$ with respect to the (020), (021) and (110) planes of PBS, respectively (Wu, Huang, Zeng, Li, \& Wang, 2014b; Zeng, et al., 2012). The locations of the three peaks did not change after incorporation of CNC nanoparticles, indicating that addition of CNC did not change the crystal structure of PBS, which was in accordance with some other nanofiller such as carbon nanotube filled PBS nanocomposite (Song \& Qiu, 2009, 2011). 


\subsection{Mechanical properties}

Tensile testing was performed to study the effect of CNC nanoparticles with small addition on the mechanical properties of PBS. Fig. 6a shows the stress-strain curves of neat PBS and PBS/CNC nanocomposites. Neat PBS showed an obvious yielding and a stable necking. The yielding strength was $25.4 \pm 0.8 \mathrm{MPa}$. Before breaking, the maximum strain was $236 \pm 20 \%$. After incorporation of $\mathrm{CNC}$, all samples exhibited apparent yielding and stable necking except PBS/CNC-1.0, which showed a fracture behavior, i.e., breaking immediately after yielding.
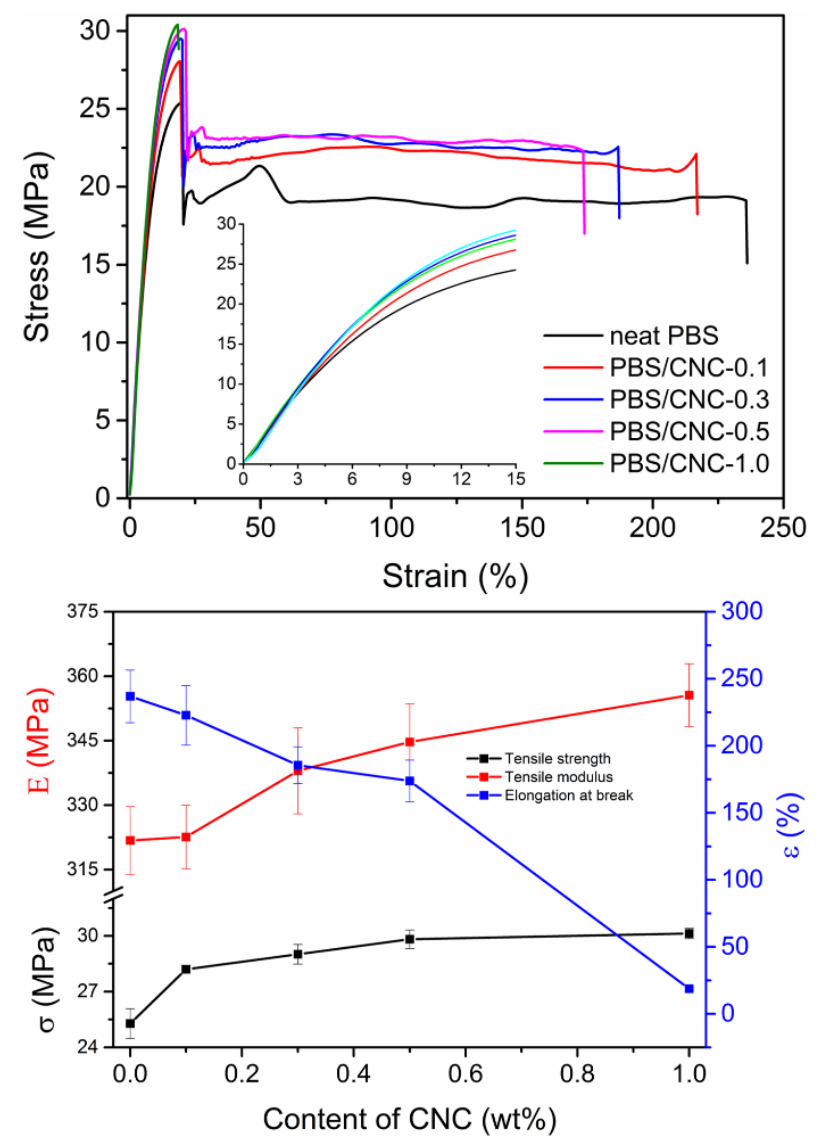

Fig. 6. Stress-strain curves of neat PBS and PBS/CNC nanocomposites (a) and tensile strength $(\sigma)$, tensile modulus $(\mathrm{E})$ and elongation at break $(\varepsilon)$ as a function of $\mathrm{CNC}$ content (b). 
It was interesting to find that the yield strength of PBS/CNC nanocomposite was enhanced compared to neat PBS, indicating reinforced mechanical properties. The yield strength increased with increasing CNC content, and the values were $28.0 \pm 0.2$, $29.5 \pm 0.5, \quad 30.1 \pm 0.5$ and $30.4 \pm 0.3 \mathrm{MPa}$ for $\mathrm{PBS} / \mathrm{CNC}-0.1, \quad \mathrm{PBS} / \mathrm{CNC}-0.3$, PNS/CNC-0.5 and PBS/CNC-1.0, respectively. The elongation at break decreased gradually with increasing $\mathrm{CNC}$ content. The values were $217 \pm 22,187 \pm 14$ and $173 \pm 16 \%$ for PBS/CNC-0.1, PBS/CNC-0.3 and PNS/CNC-0.5, respectively. When the content of $\mathrm{CNC}$ increased to $1.0 \mathrm{wt} \%$, the elongation at break dropped drastically to $18 \pm 1 \%$. It is observed from the insert of Fig. 6 a that the slope of the initial stage of stress-strain curve increased with increasing $\mathrm{CNC}$ content, indicating an increased tensile modulus, which was also an evidence for reinforcement of PBS by incorporation of CNC nanoparticles. The effect of CNC content on the tensile strength $(\sigma)$, tensile modulus (E) and elongation at break $(\varepsilon)$ of the nanocomposites is summarized in Fig. 6b. The tensile strength equals to yield strength since no strain hardening occurred before break of all the samples. The tensile modulus of PBS/CNC nanocomposite was higher than that of neat PBS and increased with increasing CNC content. The value for neat PBS was $321.8 \pm 7.9 \mathrm{MPa}$, and was improved by up to $10.5 \%$ with addition of $1.0 \mathrm{wt} \% \mathrm{CNC}$.

\section{Conclusions}

Nanocomposites consisting of PBS matrix and small amount of well dispersed CNC 
nanoparticles were successfully fabricated by a solution coagulation method. The dispersed CNC and PBS matrix showed good interfacial adhesion as evidenced by the absence of pulling out of $\mathrm{CNC}$, the increased storage modulus and complex viscosity at low frequency, as well as the slightly increased glass transition temperature. PBS/CNC nanocomposites showed double melting peaks with one lower than $100{ }^{\circ} \mathrm{C}$ and the other higher than $100{ }^{\circ} \mathrm{C}$. The low meting temperature increased while the high one kept unchanged with increasing CNC content. Melt-recrystallization mechanism accounted for the low melting peak which comes out when the melting rate overwhelms the crystallization rate. The overall isothermal crystallization kinetics was enhanced significantly with addition of small amount of $\mathrm{CNC}$, due to the significantly enhanced nucleation density. The crystallization rates of PBS were improved by 5.6, 8.2, 10.3 and 14.5 times after incorporation of $0.1,0.3,0.5$ and 1.0 wt\% CNC, respectively. The addition of CNC did not change the isothermal crystallization mechanism and crystal structure of PBS. Both tensile strength and modulus of PBS were obviously reinforced by incorporation of $\mathrm{CNC}$, while the elongation at break of PBS decreased apparently. When the content of CNC increased to $1.0 \mathrm{wt} \%$, the fracture behavior changed from ductile to brittle. In summary, PBS/CNC nanocomposites containing 0.5 wt $\%$ well dispersed CNC nanoparticles showed the optimal comprehensive properties including fast crystallization, balanced mechanical strength and ductility.

\section{Acknowledgements}


This work was supported by National Science Foundation of China (51373107) and Fundamental Research Funds for the Central Universities (SWU115006).

\section{References}

Avrami, M. (1939). Kinetics of Phase Change. I. General Theory. Journal of Chemical Physics, 7(12), 1103-1112.

Du, A.-K., Yang, K.-L., Zhao, T.-H., Wang, M., \& Zeng, J.-B. (2016). Poly(sodium 4-styrenesulfonate) wrapped carbon nanotube with low percolation threshold in poly(epsilon-caprolactone) nanocomposites. Polymer Testing, 51, 40-48.

Fortunati, E., Armentano, I., Zhou, Q., Puglia, D., Terenzi, A., Berglund, L. A., \& Kenny, J. M. (2012). Microstructure and nonisothermal cold crystallization of PLA composites based on silver nanoparticles and nanocrystalline cellulose. Polymer Degradation and Stability, 97(10), 2027-2036.

Fortunati, E., Peltzer, M., Armentano, I., Torre, L., Jimenez, A., \& Kenny, J. M. (2012). Effects of modified cellulose nanocrystals on the barrier and migration properties of PLA nano-biocomposites. Carbohydrate Polymers, 90(2), 948-956.

Fortunati, E., Puglia, D., Luzi, F., Santulli, C., Kenny, J. M., \& Torre, L. (2013). Binary PVA bio-nanocomposites containing cellulose nanocrystals extracted from different natural sources: Part I. Carbohydrate Polymers, 97(2), 825-836.

Gao, C., Zhang, S., Wang, F., Wen, B., Han, C., Ding, Y., \& Yang, M. (2014). Graphene networks with low percolation threshold in ABS nanocomposites: 
selective localization and electrical and rheological properties. ACS Applied Materials and Interfaces, 6(15), 12252-12260.

Gao, Z., Peng, J., Zhong, T., Sun, J., Wang, X., \& Yue, C. (2012). Biocompatible elastomer of waterborne polyurethane based on castor oil and polyethylene glycol with cellulose nanocrystals. Carbohydrate Polymers, 87(3), 2068-2075.

Habibi, Y., Lucia, L. A., \& Rojas, O. J. (2010). Cellulose Nanocrystals: Chemistry, Self-Assembly, and Applications. Chemical Reviews, 110(6), 3479-3500.

Hu, F., Lin, N., Chang, P. R., \& Huang, J. (2015). Reinforcement and nucleation of acetylated cellulose nanocrystals in foamed polyester composites. Carbohydrate Polymers, 129, 208-215.

Huang, H.-D., Xu, J.-Z., Fan, Y., Xu, L., \& Li, Z.-M. (2013). Poly(l-lactic acid) Crystallization in a Confined Space Containing Graphene Oxide Nanosheets. The Journal of Physical Chemistry B, 117(36), 10641-10651.

Kamal, M. R., \& Khoshkava, V. (2015). Effect of cellulose nanocrystals (CNC) on rheological and mechanical properties and crystallization behavior of PLA/CNC nanocomposites. Carbohydrate Polymers, 123, 105-114.

Lin, N., Chen, Y., Hu, F., \& Huang, J. (2015). Mechanical reinforcement of cellulose nanocrystals on biodegradable microcellular foams with melt-compounding process. Cellulose, 22(4), 2629-2639.

Lin, N., Huang, J., Chang, P. R., Feng, J., \& Yu, J. (2011). Surface acetylation of cellulose nanocrystal and its reinforcing function in poly(lactic acid). Carbohydrate Polymers, 83(4), 1834-1842. 
Lin, N., Yu, J., Chang, P. R., Li, J., \& Huang, J. (2011). Poly(butylene succinate)-Based Biocomposites Filled With Polysaccharide Nanocrystals: Structure and Properties. Polymer Composites, 32(3), 472-482.

Liu, G.-C., Zeng, J.-B., Huang, C.-L., Jiao, L., Wang, X.-L., \& Wang, Y.-Z. (2013). Crystallization Kinetics and Spherulitic Morphologies of Biodegradable Poly(butylene succinate-co-diethylene glycol succinate) Copolymers. Industrial \& Engineering Chemistry Research, 52(4), 1591-1599.

Lu, X., Zeng, J.-B., Huang, C.-L., \& Wang, Y.-Z. (2012). Isothermal Crystallization Behavior of Biodegradable P(BS-b-PEGS) Multiblock Copolymers. Industrial \& Engineering Chemistry Research, 51(24), 8262-8272.

Mariano, M., El Kissi, N., \& Dufresne, A. (2016). Cellulose nanocrystal reinforced oxidized natural rubber nanocomposites. Carbohydrate Polymers, 137, 174-183.

Miao, C., \& Hamad, W. Y. (2016). In-situ polymerized cellulose nanocrystals (CNC) poly(L-lactide) (PLLA) nanomaterials and applications in nanocomposite processing. Carbohydrate Polymers, 153, 549-558.

Mishra, J. K., Hwang, K.-J., \& Ha, C.-S. (2005). Preparation, mechanical and rheological properties of a thermoplastic polyolefin (TPO)/organoclay nanocomposite with reference to the effect of maleic anhydride modified polypropylene as a compatibilizer. Polymer, 46(6), 1995-2002.

Pötschke, P., Fornes, T. D., \& Paul, D. R. (2002). Rheological behavior of multiwalled carbon nanotube/polycarbonate composites. Polymer, 43(11), 3247-3255. 
Paralikara, S. A., Simonsen, J., \& Lombardi, J. (2008). Poly(vinyl alcohol)/cellulose nanocrystal barrier membranes. Journal of Membrane Science, 320(1-2), $248-258$.

Qiu, Z., Komura, M., Ikehara, T., \& Nishi, T. (2003). DSC and TMDSC study of melting behaviour of poly(butylene succinate) and poly(ethylene succinate). Polymer, 44(26), 7781-7785.

Samir, M., Alloin, F., Sanchez, J. Y., El Kissi, N., \& Dufresne, A. (2004). Preparation of cellulose whiskers reinforced nanocomposites from an organic medium suspension. Macromolecules, 37(4), 1386-1393.

Santamaria-Echart, A., Ugarte, L., Garcia-Astrain, C., Arbelaiz, A., Angeles Corcuera, M., \& Eceiza, A. (2016). Cellulose nanocrystals reinforced environmentally-friendly waterborne polyurethane nanocomposites. Carbohydrate Polymers, 151, 1203-1209.

Song, L., \& Qiu, Z. (2009). Crystallization behavior and thermal property of biodegradable poly(butylene succinate)/functional multi-walled carbon nanotubes nanocomposite. Polymer Degradation and Stability, 94(4), $632-637$.

Song, L., \& Qiu, Z. (2011). Influence of low multi-walled carbon nanotubes loadings on the crystallization behavior of biodegradable poly(butylene succinate) nanocomposites. Polymers for Advanced Technologies, 22(12), 1642-1649.

Wang, M., Deng, X.-Y., Du, A.-K., Zhao, T.-H., \& Zeng, J.-B. (2015). Poly(sodium 4-styrenesulfonate) modified graphene for reinforced biodegradable 
poly(epsilon-caprolactone) nanocomposites. RSC Advances, 5(89), $73146-73154$

Wang, S., Lu, A., \& Zhang, L. (2016). Recent advances in regenerated cellulose materials. Progress in Polymer Science, 53, 169-206.

Wu, F., Huang, C.-L., Zeng, J.-B., Li, S.-L., \& Wang, Y.-Z. (2014a). Composition dependence of physical properties of biodegradable poly(ethylene succinate) urethane ionenes. RSC Advances, 4(97), 54175-54186.

Wu, F., Huang, C.-L., Zeng, J.-B., Li, S.-L., \& Wang, Y.-Z. (2014b). Synthesis and characterization of segmented poly(butylene succinate) urethane ionenes containing secondary amine cation. Polymer, 55(16), 4358-4368.

Xu, J.-Z., Chen, T., Yang, C.-L., Li, Z.-M., Mao, Y.-M., Zeng, B.-Q., \& Hsiao, B. S. (2010). Isothermal Crystallization of Poly(L-lactide) Induced by Graphene Nanosheets and Carbon Nanotubes: A Comparative Study. Macromolecules, 43(11), 5000-5008.

Yasuniwa, M., \& Satou, T. (2002). Multiple melting behavior of poly(butylene succinate). I. Thermal analysis of melt-crystallized samples. Journal of Polymer Science Part B-Polymer Physics, 40(21), 2411-2420.

Yasuniwa, M., Tsubakihara, S., Ohoshita, K., \& Tokudome, S. (2001). X-ray studies on the double melting behavior of poly(butylene terephthalate). Journal of Polymer Science Part B-Polymer Physics, 39(17), 2005-2015.

Yasuniwa, M., Tsubakihara, S., Satou, T., \& Iura, K. (2005). Multiple melting behavior of poly(butylene succinate). II. Thermal analysis of isothermal 
crystallization and melting process. Journal of Polymer Science Part B-Polymer Physics, 43(15), 2039-2047.

Yu, H., Yan, C., \& Yao, J. (2014). Fully biodegradable food packaging materials based on functionalized cellulose nanocrystals/poly(3-hydroxybutyrate-co-3-hydroxyvalerate) nanocomposites. RSC Advances, 4(104), 59792-59802.

Yuan, L., Wu, D., Zhang, M., Zhou, W., \& Lin, D. (2011). Rheological percolation behavior and isothermal crystallization of poly(butylene succinate)/carbon nanotube composites. Industrial Engineering Chemistry Research, 50(24), 14186-14192.

Zeng, J.-B., He, Y.-S., Li, S.-L., \& Wang, Y.-Z. (2012). Chitin Whiskers: An Overview. Biomacromolecules, 13(1), 1-11.

Zeng, J.-B., Huang, C.-L., Jiao, L., Lu, X., Wang, Y.-Z., \& Wang, X.-L. (2012). Synthesis and Properties of Biodegradable Poly(butylene succinate-co-diethylene glycol succinate) Copolymers. Industrial \& Engineering Chemistry Research, 51(38), 12258-12265.

Zeng, J.-B., Jiao, L., Li, Y.-D., Srinivasan, M., Li, T., \& Wang, Y.-Z. (2011). Bio-based blends of starch and poly(butylene succinate) with improved miscibility, mechanical properties, and reduced water absorption. Carbohydrate Polymers, 83(2), 762-768.

Zeng, J.-B., Srinivansan, M., Li, S.-L., Narayan, R., \& Wang, Y.-Z. (2011). Nonisothermal and Isothermal Cold Crystallization Behaviors of 
Biodegradable Poly(p-dioxanone). Industrial \& Engineering Chemistry Research, 50(8), 4471-4477.

Zeng, J.-B., Zhu, Q.-Y., Li, Y.-D., Qiu, Z.-C., \& Wang, Y.-Z. (2010). Unique Crystalline/Crystalline Polymer Blends of Poly(ethylene succinate) and Poly(p-dioxanone): Miscibility and Crystallization Behaviors. Journal of Physical Chemistry B, 114(46), 14827-14833.

Zeng, R. T., Hu, W., Wang, M., Zhang, S.-D., \& Zeng, J.-B. (2016). Morphology, rheological and crystallization behavior in non-covalently functionalized carbon nanotube reinforced poly(butylene succinate) nanocomposites with low percolation threshold. Polymer Testing, 50, 182-190.

Zhang, Y. R., Wang, X. L., Zhao, G. M., \& Wang, Y. Z. (2012). Preparation and properties of oxidized starch with high degree of oxidation. Carbohydrate Polymers, 87(4), 2554-2562.

Zhang, Y. R., Zhang, S. D., Wang, X. L., Chen, R. Y., \& Wang, Y. Z. (2009). Effect of carbonyl content on the properties of thermoplastic oxidized starch. Carbohydrate Polymers, 78(1), 157-161.

Zhao, T.-H., Yang, K.-L., Zeng, R.-T., Du, A.-K., Wang, M., \& Zeng, J.-B. (2016). Crystallization Behavior of Poly(sodium 4-styrenesulfonate)-Functionalized Carbon Nanotubes Filled Poly(epsilon-caprolactone) Nanocomposites. Industrial \& Engineering Chemistry Research, 55(7), 1881-1889.

Zhou, L., He, H., Jiang, C., \& He, S. (2015). Preparation and characterization of poly(glycerol sebacate)/cellulose nanocrystals elastomeric composites. 
Journal of Applied Polymer Science, 132(27), 42196.

Zhu, J., Dong, X. T., Wang, X. L., \& Wang, Y. Z. (2010). Preparation and properties of a novel biodegradable ethyl cellulose grafting copolymer with poly(p-dioxanone) side-chains. Carbohydrate Polymers, 80(2), 350-359. 